\title{
Orthodontic with Miniplates in the Correction of an Adult Case with Class II Malocclusion and Anterior Open Bite
}

\author{
Ali S Aljhani ${ }^{1 *}$, Maryam Alhindi ${ }^{2}$ and Khalid H Zawawi ${ }^{3}$ \\ ${ }^{1}$ King Saud Bin Abdulaziz University for Health Sciences, Saudi Arabia \\ ${ }^{2}$ Dental College, King Saud University, Saudi Arabia \\ ${ }^{3}$ Department of Orthodontics, Dental College, King Abdulaziz University, Saudi Arabia
}

*Corresponding author: Dr. Ali S Aljhani, BDS, MSD, ABO, Orthodontic Consultant, KAMC, Assistant Professor, King Saud Bin Abdulaziz University for Health Sciences, Saudi Arabia

\begin{abstract}
This case report illustrates an orthodontic treatment of a 23-year-old female patient presented with an Angle Class II malocclusion, open bite, bimaxillary dental protrusion and incompetent lips with the aid of titanium miniplates. After extraction of her maxillary first bicuspids and mandibular second bicuspids, fixed pre-adjusted bi-dimensional Edgewise appliances were placed. After alignment, titanium miniplates were surgically placed into the zygomatic buttresses bilaterally. Anterior retraction and intrusion, as well as posterior intrusion were performed. Treatment time was 20 months. Cephalometric superimpositions showed favourable maxillary molar intrusion and mandibular autorotation. Open bite was corrected and class I molars and canines with ideal overjet were achieved with a significant improvement on appearance. Orthodontic treatment with miniplates is an effective technique in open bite cases with vertical problems.
\end{abstract}

\section{Keywords}

Temporary anchorage devices, Miniplates, Orthodontic tooth movement, Intrusion, Bimaxillary protrusion

\section{Introduction}

One of the most challenging orthodontic problems is anterior open bite [1,2]. Conventional treatment for the correction of open bite includes the use of high-pull headgear, tongue cribs/spurs, posterior bite blocks, posterior magnets, fixed appliances such as the Multiloop Edgewise Arch Wire (MEAW), and the use of vertical elastics [3-6]. All these techniques depend on patient compliance and are less successful in adults.

Since the introduction of Temporary Skeletal An- chorage Devices (TADs), new possibilities for diverse treatment options to orthodontic treatment were introduced [7-11]. These anchorage devices permit the application of skeletal anchorage techniques which can be superior to traditional orthodontic mechanics in various situations, since patient compliance is not required, and force application is possible in different directions without untoward effects [9].

Several different temporary skeletal anchorage systems exist. The most widely used anchorage systems are miniscrews and miniplates. Even though miniscrews are more used and their reported success rate is approximately $86.5 \%$, miniplates have a slightly better success rate of $91-96 \%$ [12-15]. This could be due to the tendency of miniscrews to become loose during orthodontic force application. However, the ease of miniscrews insertion and removal under local anesthesia, usually by the orthodontist, gives them an advantage over miniplates, which require an oral surgeon to carry out the procedure. Nevertheless, certain clinical situations exist where miniplates are preferable.

Traditionally, severe anterior open bite cases have required orthognathic surgical intervention. Alternatively, a number of reports have successfully demonstrated the treatment of open bite with miniscrews [1618].

This case report shows the treatment of a Class II open bite case with a bimaxillary dental protrusion and lip incompetence using maxillary miniplates.

Citation: Aljhani AS, Alhindi M, Zawawi KH (2018) Orthodontic with Miniplates in the Correction of an Adult Case with Class II Malocclusion and Anterior Open Bite. Int J Oral Dent Health 4:059. doi. org/10.23937/2469-5734/1510059

Accepted: September 01, 2018: Published: September 03, 2018

Copyright: (c) 2018 Aljhani AS, et al. This is an open-access article distributed under the terms of the Creative Commons Attribution License, which permits unrestricted use, distribution, and reproduction in any medium, provided the original author and source are credited. 

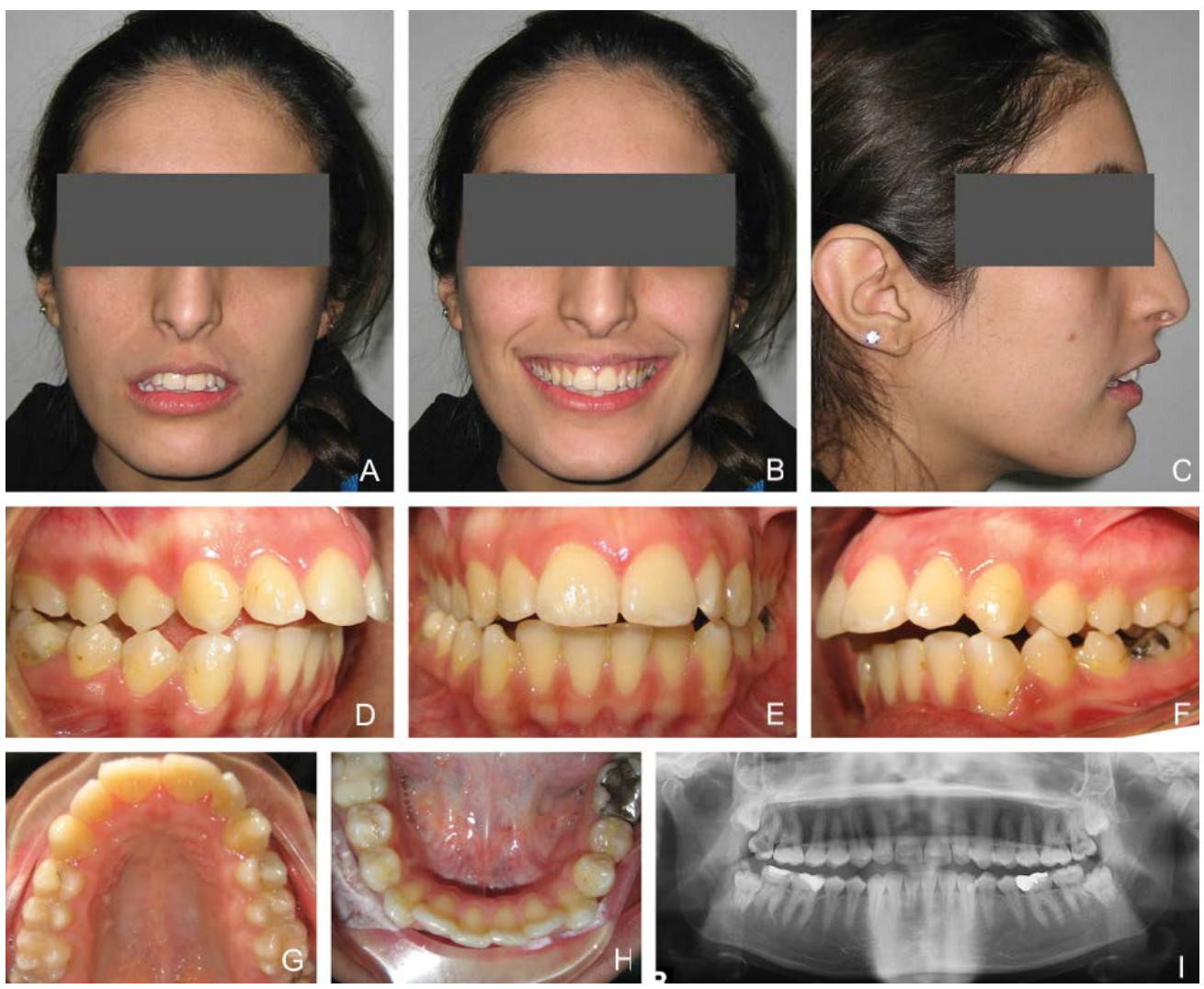

Figure 1: Pre-treatment records: A) Extra-oral: Frontal at rest; B) Extra-oral: Frontal at smile; C) Extra-oral: Profile at rest; D) Intra-oral: Right; E) Intra-oral: Frontal; F) Intra-oral: left; G) Intra-oral: Occlusal upper; H) Intra-oral: Occlusal lower; I) Panoramic x-ray.

Table 1: Pre-and post-treatment cephalometric analysis.

\begin{tabular}{|c|c|c|c|}
\hline Measurement & Norms & Pre-treatment & Post-treatment \\
\hline Inclination of $\mathrm{SN}$ to $\mathrm{FH}$ & $7^{\circ}$ & $14^{\circ}$ & $13.9^{\circ}$ \\
\hline $\operatorname{SNA}\left({ }^{\circ}\right)$ & $82^{\circ}$ & $76.9^{\circ}$ & $76.1^{\circ}$ \\
\hline SNB $\left({ }^{\circ}\right)$ & $80^{\circ}$ & $72.7^{\circ}$ & $72.8^{\circ}$ \\
\hline ANB $\left({ }^{\circ}\right)$ & $2^{\circ}$ & $4.1^{\circ}$ & $3.3^{\circ}$ \\
\hline Wits appraisal (mm) & $0^{\circ}$ & $4.3 \mathrm{~mm}$ & $2.4 \mathrm{~mm}$ \\
\hline Facial angle $(\mathrm{NPg}-\mathrm{FH})\left(^{\circ}\right)$ & $87.8^{\circ}$ & $83.9^{\circ}$ & $82.5^{\circ}$ \\
\hline Angle of convexity (NA-APg) $\left(^{\circ}\right)$ & $0^{\circ}$ & $2.2^{\circ}$ & $1.0^{\circ}$ \\
\hline Mandibular plane (SN - GoGn) $\left(^{\circ}\right)$ & $32.9^{\circ}$ & $36^{\circ}$ & $35.1^{\circ}$ \\
\hline LFH/TFH (ANS-Me/N-Me) \% & $55 \%$ & $57.20 \%$ & $56.30 \%$ \\
\hline Occlusal plan-SN & $14^{\circ}$ & $16.9^{\circ}$ & $18.9^{\circ}$ \\
\hline U1-Palatal plane (ANS-PNS) & $110^{\circ}$ & $113.1^{\circ}$ & $99.9^{\circ}$ \\
\hline U1-NA $\left({ }^{\circ}\right)$ & $22^{\circ}$ & $32^{\circ}$ & $18.5^{\circ}$ \\
\hline U1-NA (mm) & $4 \mathrm{~mm}$ & $7.4 \mathrm{~mm}$ & $1.0 \mathrm{~mm}$ \\
\hline L1-NB $\left({ }^{\circ}\right)$ & $25^{\circ}$ & $27.9^{\circ}$ & $18.4^{\circ}$ \\
\hline L1-NB (mm) & $4 \mathrm{~mm}$ & $5.5 \mathrm{~mm}$ & $1.8 \mathrm{~mm}$ \\
\hline FMA $\left(^{\circ}\right)$ & $25^{\circ}$ & 27.9 & 31.3 \\
\hline FMIA $\left({ }^{\circ}\right)$ & $65^{\circ}$ & 53 & 61.7 \\
\hline $\operatorname{IMPA}\left({ }^{\circ}\right)$ & $90^{\circ}$ & $99.1^{\circ}$ & $89.5^{\circ}$ \\
\hline Nasolabial angle $\left({ }^{\circ}\right)$ & $90-110^{\circ}$ & $106^{\circ}$ & $117.3^{\circ}$ \\
\hline Upper lip to esthetic plane & $-4 \mathrm{~mm}$ & $-3.7 \mathrm{~mm}$ & $-7.1 \mathrm{~mm}$ \\
\hline Lower lip to esthetic plane & $-2 \mathrm{~mm}$ & $-4.2 \mathrm{~mm}$ & $-6.7 \mathrm{~mm}$ \\
\hline
\end{tabular}

SN: sella nasion plan; FH: frankfort horizontal; A: point A; B: point B; Pg: pogonion; LFH: lower face height; TFH: total face height; ANS: anterior nasal spine; PNS: posterior nasal spine; Me: menton; U1: upper central incisor; L1: lower central incisor; FMA: frankfort mandibular angle; FMIA: frankfort mandibular incisor angle; IMPA: incisor mandibular plane angle.

\section{Case Report}

\section{Case summary}

A 23-year-old female presented with a chief complaint of "I don't like my smile and my teeth are stick- ing out". Her medical history was non-contributory. Her dental history showed multiple restorations, gingivitis, and poor oral hygiene. No signs or symptoms of temporomandibular dysfunction were noted. She had a convex profile, slightly acute nasolabial angle, and pro- 
trusive lower lip. At smiling, she displays $100 \%$ of the maxillary teeth and adequate amount of gingiva. At rest, she had incompetent lips with $75 \%$ tooth show (Figure 1). Dentally, she had bilateral Class II molar relationship (4 mm), Class II canine relationship ( $3 \mathrm{~mm}$ bilaterally) with $7 \mathrm{~mm}$ overjet and mild anterior open bite. Upper and lower anterior teeth were proclined and protruded. There was $1 \mathrm{~mm}$ crowding in the upper arch and $5 \mathrm{~mm}$ in the lower arch. Upper midline was coincident with the facial midline, while the lower was shifted $2 \mathrm{~mm}$ to the right. The upper arch form was ovoid, and the lower arch form was square, which led to a mild transverse discrepancy. No centric relation/centric occlusion shift was detected.

The lateral cephalometric analysis indicated a mild Class II skeletal relationship, a hyperdivergent mandibular plane angle, and proclined and protruded bimaxillary incisors (Figure 2 and Table 1).

Diagnosis: The patient was diagnosed as having an Angle Class II malocclusion with Class II skeletal pattern; Bimaxillary protrusion; Hyperdivergent mandibular plane; Incompetent lips and an open bite.

Treatment objective: The main objective was to achieve class I molar and canine relationship, achieve ideal overjet and overbite, avoid tipping of the incisors to avoid causing gummy smile, intruding the posterior teeth to allow for counter-clockwise rotation of the mandible, and coordinate the upper and lower arch forms. The facial aesthetics necessitated reducing the

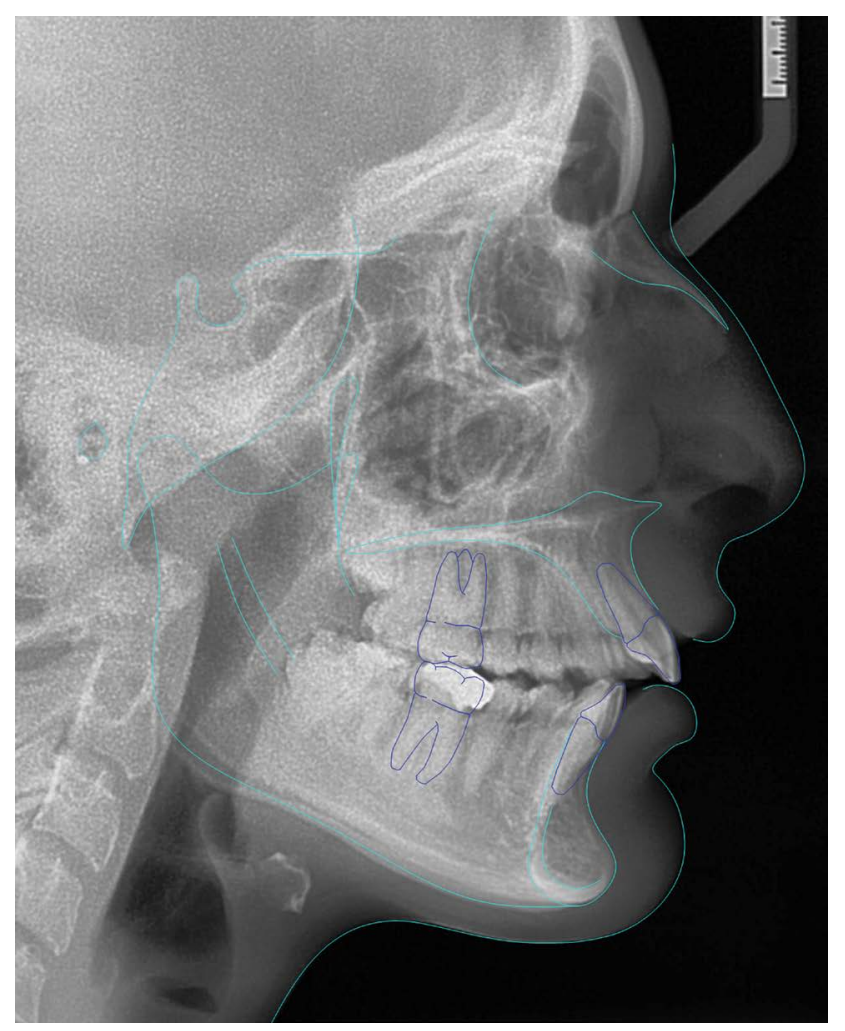

Figure 2: Pre-treatment cephalometric tracing showing mild Class II skeletal relationship, a hyperdivergent mandibular plane angle and proclined and protruded bimaxillary incisors. soft tissue protrusion and improve the inter-labial gap. Maximum anchorage of the maxillary molars was also needed to reduce the overjet to achieve class I canine relationship.

Treatment alternatives: The patient was given two options, both including extraction of maxillary first bicuspids and mandibular second bicuspids, to reduce the dental protrusion and alleviate crowding. The first option was surgical, where the maxilla will be impacted, more posterior than anterior, with mandibular advancement. The second option was the usage of temporary skeletal anchorage device to reduce the protrusion and impact the maxilla to allow for counter-clockwise rotation of the mandible.

Treatment progress: The patient was referred to extract the maxillary first bicuspids and mandibular second bicuspids. Fixed pre-adjusted bi-dimensional Edgewise appliances were placed $(0.018 \times 0.025$ slot in the incisors and $0.022 \times 0.028$ slot in the canines, bicuspids, and molars). A transpalatal arch was also cemented to prevent buccal flaring of the molars during the intrusion phase. The patient was then referred to the oral surgeon to place the miniplates. Under local anaesthesia, gingival mucoperiosteal flap was raised exposing the infrazygomatic crest. Titanium miniplates (Dentsply, Sankin, Tokyo, Japan) were fixed at the zygomatic buttresses bilaterally with titanium screws. After a healing period of 4 weeks, $0.018 \times 0.022$ Stainless Steel (SS) arch wire was inserted in the upper arch and en masse anterior retraction was started with elastic chain modules to the miniplates (Figure 3). Careful consideration was applied in force application to achieve anterior intrusion. Posterior intrusion was started at the same time with light force. Elastic power chain modules were used to close the extraction spaces from the hook of the titanium miniplate to a hook on the arch wire with an initial force of approximately $200 \mathrm{~g}$ per side.

Space closure in the lower arch was also accomplished using elastic power chain modules on $0.018 \times$ 0.022 SS arch wire with minimal anchorage requirements. Once the desired occlusion was achieved, the fixed appliances and titanium miniplates were removed. Bonded retainers were applied to the upper and lower anterior teeth and an upper wraparound removable retainer was delivered (Figure 4).

Treatment results: A well-balanced profile was achieved with competent lips, slightly obtuse nasolabial angle with a relatively prominent chin. The gum display was improved with proper incisal show. Dentally, class I molar and canine relationships were achieved bilaterally. Ideal overjet and over bite with well-coordinated arches were also achieved. Maxillary and mandibular dental midlines are coincident with each other and with the facial midline (Figure 5).

Cephalometric superimpositions before and after treatment showed retraction of the maxillary anterior 

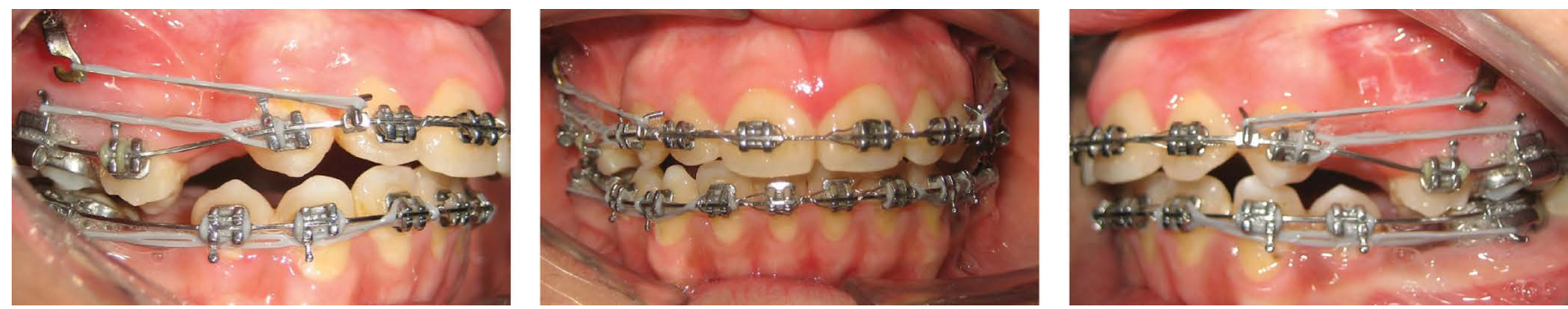

Figure 3: En masse anterior retraction.
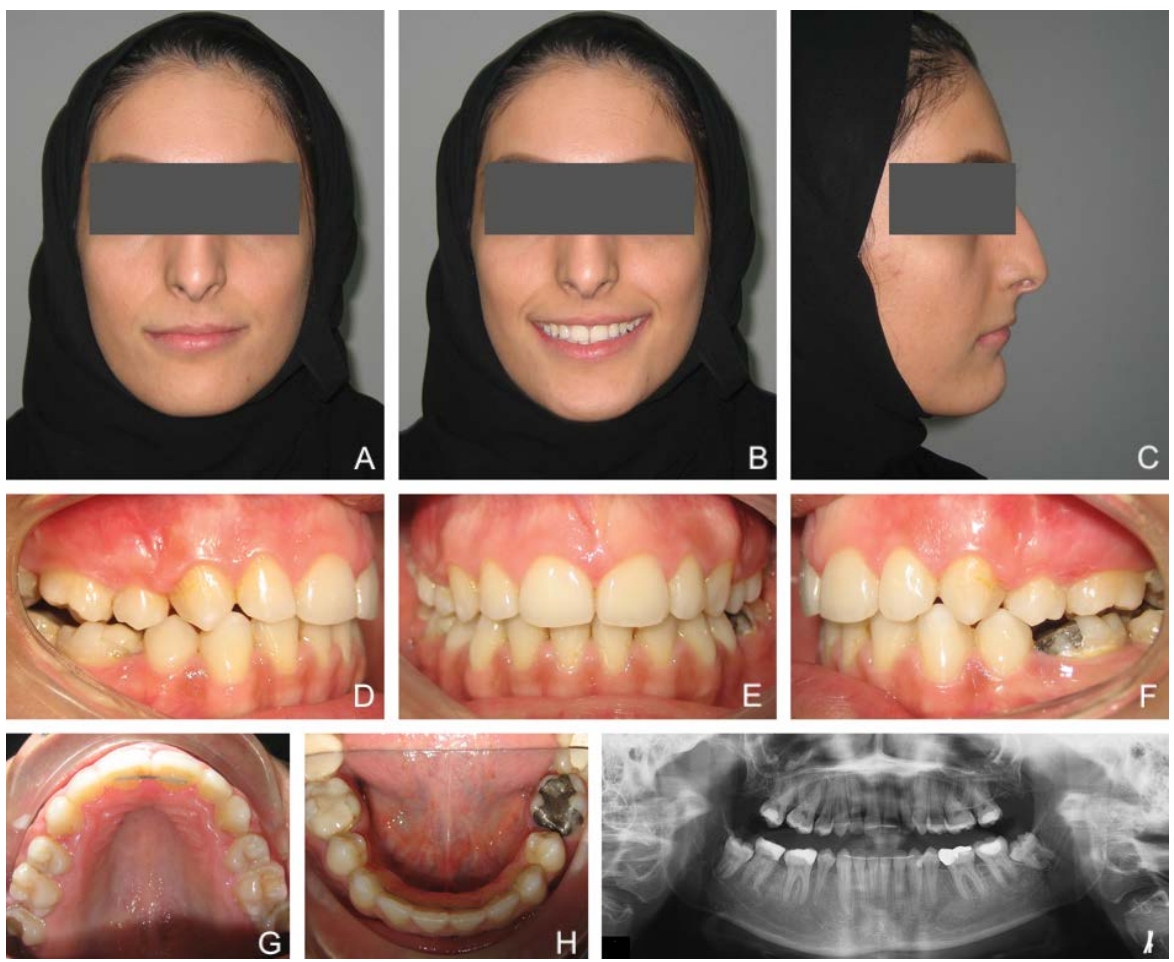

Figure 4: Post-treatment records: A) Extra-oral: Frontal at rest; B) Extra-oral: Frontal at smile; C) Extra-oral: Profile at rest; D) Intra-oral: Right; E) Intra-oral: Frontal; F) Intra-oral: left; G) Intra-oral: Occlusal upper; H) Intra-oral: Occlusal lower; I) Panoramic x-ray. Bonded retainers were applied to the upper and lower anterior teeth and an upper wrap around removable retainer were delivered.

teeth with intrusion of the maxillary molars and no loss of anchorage. Mandibular anterior teeth were tipped back but remained in their position and molars protracted to Class I occlusion. Counter-clockwise rotation to the mandible was also achieved (Figure 6).

During the active treatment, no significant periodontal problems, such as gingival recession or loss of tooth vitality, and no looseness or deformation of the titanium miniplates were observed.

\section{Discussion}

The development of skeletal anchorage has allowed the development of innovative orthodontic treatment techniques. Difficult treatments became less complicated and more predictable. Also, treatment duration has decreased. The need for orthognathic surgery has reduced and sometimes avoided. These results couldn't have been achieved without the aid of skeletal anchorage devices, namely miniscrews and miniplates $[2,15]$.

The advantages of miniplates over miniscrews are due to their better stability and that the screw insertion is beyond tooth apices, allowing anteroposterior and vertical movement of adjacent teeth. Miniplates are mostly suggested in situations needing the application of heavy orthodontic forces or the combined movement of several teeth, as they do not interfere with tooth movement and allow teeth close to the miniplates to be moved [20-22].

This article demonstrates the successful treatment of a 23-year-old female with Class II malocclusion, open bite, bimaxillary protrusion and incompetent lips treated with titanium miniplates. The intrusion of molars to correct anterior open bite was successfully achieved and the soft tissue profile was also improved by the autorotation of the mandible. Class I molar and canine relationships were attained with the aid of miniplates.

Although patients who receive flap surgery for the placement of titanium miniplates more frequently complain of both swelling and pain than those patients with titanium screws, the titanium miniplates have advan- 


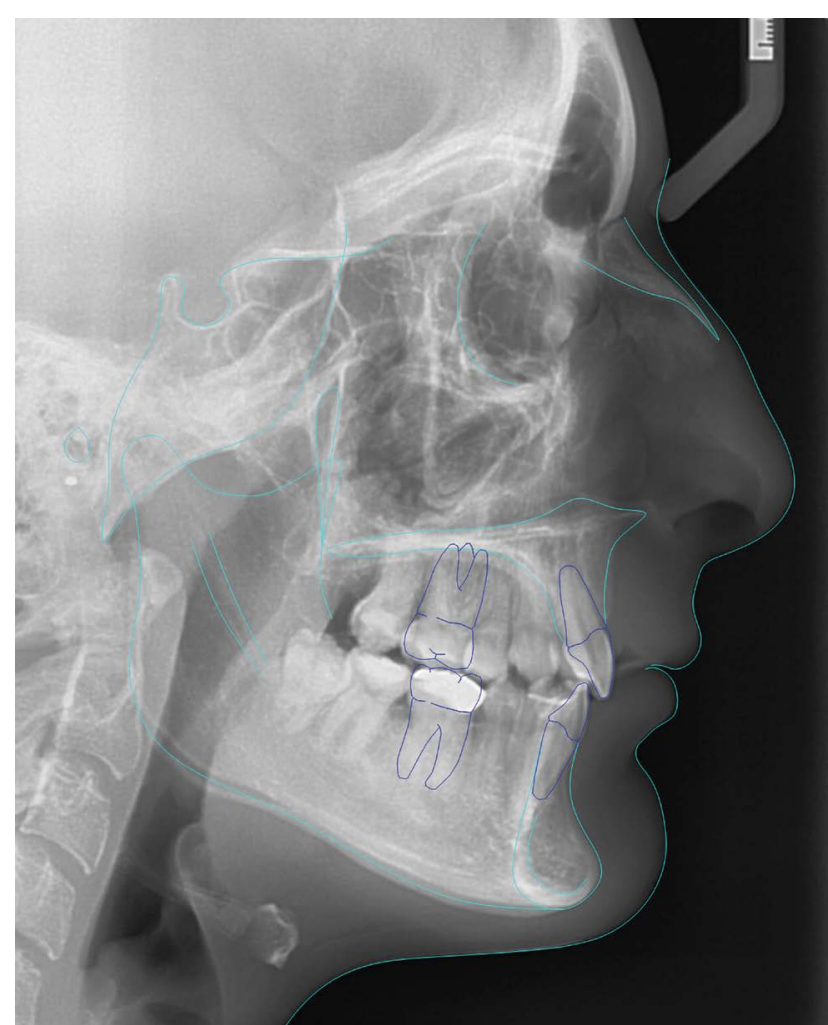

Figure 5: Post-treatment cephalometric tracing.

tages such as a strong resistance to force and a nearly $100 \%$ success rate $[12,16]$. Therefore, in this present case, titanium miniplates were used as TADs, since a strong force might have been needed when retracting anterior teeth after the corticotomy.

Furthermore, anchorage loss often produces insufficient treatment results, particularly in patients who require maximum anchorage, and such treatment further produces the extension of orthodontic treatment period. Temporary anchorage devices have been shown to provide an effective anchorage to obtain en masse retraction of anterior teeth without anchorage loss $[11,23]$. In this case, titanium miniplates were used to establish absolute anchorage [4,24]. They were necessary to retract the maxillary anterior teeth with the absolute anchorage of maxillary molars in order to improve severe upper and lower dental protrusion, and to achieve class I relationships. The extraction spaces were closed with power chains from the titanium plate to the hook on the arch wire, and the superimposition of preand post-treatment cephalometric radiographs showed no mesial movement of the maxillary molars.

Moreover, in the current case, titanium miniplates were placed on the infrazygomatic crest of the maxilla, as the bone of that region was thick and distant from the roots. Although an orthodontic force could be applied to the titanium miniplates immediately after implantation, it is advisable to wait for healing of the wound and for the patient to learn how to clean the peripheral region of the miniplates [4]. Therefore, the patient had 4 weeks of healing time after placement of the titanium
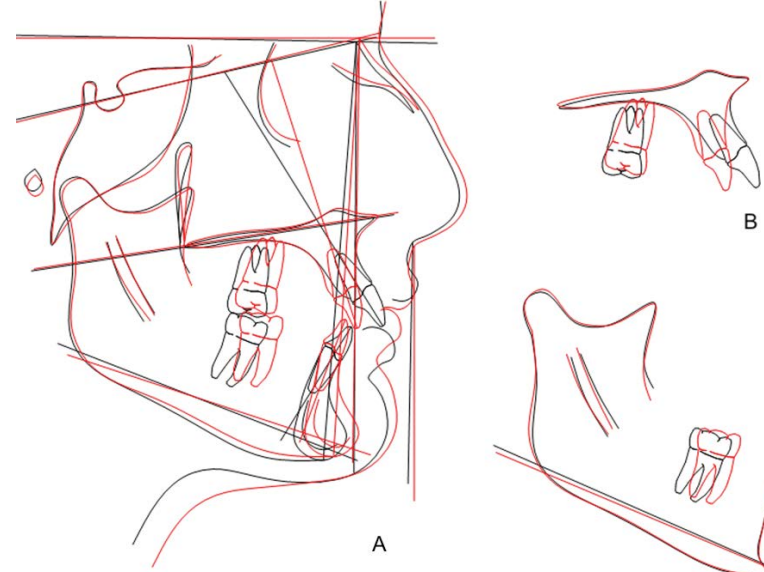

B

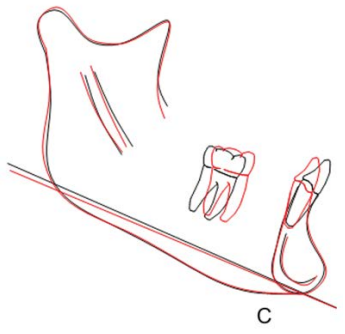

Figure 6: Cephalometric superimpositions before and after treatment: A) Counter-clockwise rotation to the mandible achieved; B) Retraction of the maxillary anterior teeth with intrusion of the maxillary molars; C) Mandibular anterior teeth were tipped back but remained in their position and molars protracted to Class I occlusion.

miniplates in the current case. It is worthy to note that during the treatment, the patient did not indicate any significant symptoms related to the miniplates.

\section{Conclusions}

Orthodontic treatment with miniplates is an effective technique in open bite cases with vertical problems. Intrusion was accomplished successfully with miniplates with no side effects. This case reports the effectiveness of titanic miniplates for absolute anchorage, especially in situations where great corrections are needed, involving sagittal and vertical problems.

\section{References}

1. Reichert I, Figel P, Winchester L (2014) Orthodontic treatment of anterior open bite: A review article--is surgery always necessary? Oral Maxillofac Surg 18: 271-277.

2. Alsafadi AS, Alabdullah MM, Saltaji $H$, Abdo A, Youssef $M$ (2016) Effect of molar intrusion with temporary anchorage devices in patients with anterior open bite: A systematic review. Prog Orthod 17: 9.

3. Lai EH, Yao CC, Chang JZ, Chen I, Chen YJ (2008) Three-dimensional dental model analysis of treatment outcomes for protrusive maxillary dentition: Comparison of headgear, miniscrew, and miniplate skeletal anchorage. Am J Orthod Dentofacial Orthop 134: 636-645.

4. Umemori M, Sugawara J, Mitani H, Nagasaka H, Kawamura H (1999) Skeletal anchorage system for open-bite correction. Am J Orthod Dentofacial Orthop 115: 166-174.

5. Albogha MH, Takahashi I, Sawan MN (2015) Early treatment of anterior open bite: Comparison of the vertical and horizontal morphological changes induced by magnetic bite-blocks and adjusted rapid molar intruders. Korean $\mathrm{J}$ Orthod 45: 38-46.

6. Saito I, Yamaki M, Hanada K (2005) Nonsurgical treatment of adult open bite using edgewise appliance combined with high-pull headgear and class III elastics. Angle Orthod 75: 277-283. 
7. Creekmore TD, Eklund MK (1983) The possibility of skeletal anchorage. J Clin Orthod 17: 266-269.

8. Kanomi R (1997) Mini-implant for orthodontic anchorage. J Clin Orthod 31: 763-767.

9. Cope J (2005) Temporary anchorage devices in orthodontics: A paradigm shift. Semin Orthod 11: 3-9.

10. Al-Fraidi AA, Zawawi KH (2010) Selective intrusion of overerupted upper first molars using a temporary anchorage device: Case report. J Can Dent Assoc 76: 25-30.

11. Aljhani A, Zawawi KH (2010) The use of mini-implants in en masse retraction for the treatment of bimaxillary dentoalveolar protrusion. Saudi Dent J 22: 35-39.

12. Lee SJ, Lin L, Kim SH, Chung KR, Donatelli RE (2013) Survival analysis of a miniplate and tube device designed to provide skeletal anchorage. Am J Orthod Dentofacial Orthop 144: 349-356.

13. Papageorgiou SN, Zogakis IP, Papadopoulos MA (2012) Failure rates and associated risk factors of orthodontic miniscrew implants: A meta-analysis. Am J Orthod Dentofacial Orthop 142: 577-595.

14. Rodriguez JC, Suarez F, Chan HL, Padial-Molina M, Wang HL (2014) Implants for orthodontic anchorage: Success rates and reasons of failures. Implant Dent 23: 155-161.

15. Tsui WK, Chua HD, Cheung LK (2012) Bone anchor systems for orthodontic application: A systematic review. Int J Oral Maxillofac Surg 41: 1427-1438.

16. Xun C, Zeng X, Wang X (2007) Microscrew anchorage in skeletal anterior open-bite treatment. Angle Orthod 77: 47-56.
17. Yanagita T, Adachi R, Kamioka H, Yamashiro T (2013) Severe open bite due to traumatic condylar fractures treated nonsurgically with implanted miniscrew anchorage. Am J Orthod Dentofacial Orthop 143: S137-S147.

18. Rachala MR, Harikrishnan P (2010) Management of severe anterior open-bite in an adult patient using miniscrews as skeletal anchorage. Int J Orthod Milwaukee 21: 9-14.

19. Sherwood KH, Burch J, Thompson W (2003) Intrusion of supererupted molars with titanium miniplate anchorage. Angle Orthod 73: 597-601.

20. Sugawara J, Daimaruya T, Umemori M, Nagasaka H, Takahashi I, et al. (2004) Distal movement of mandibular molars in adult patients with the skeletal anchorage system. Am J Orthod Dentofacial Orthop 125: 130-138.

21. Faber J, Velasque $F(2009)$ Titanium miniplate as anchorage to close a premolar space by means of mesial movement of the maxillary molars. Am J Orthod Dentofacial Orthop 136: 587-595.

22. Cheng SJ, Tseng IY, Lee JJ, Kok SH (2004) A prospective study of the risk factors associated with failure of mini-implants used for orthodontic anchorage. Int J Oral Maxillofac Implants 19: 100-106.

23. Chung KR, Kim YS, Linton JL, Lee YJ (2002) The miniplate with tube for skeletal anchorage. J Clin Orthod 36: 407-412.

24. lino S, Sakoda S, Miyawaki S (2006) An adult bimaxillary protrusion treated with corticotomy-facilitated orthodontics and titanium miniplates. Angle Orthod 76: 1074-1082. 